\title{
Nature and outcomes of the increased incidence of colorectal malignancy after liver transplantation in Australasia
} \section{Deborah J Verran
MB ChB, FRACS, Transplant Surgeon \\ Marie H Mulhearn \\ BAppSc (Nursing). GradDipHPEd, \\ Data Manager \\ Pamela J Dilworth \\ Programme Supervisor}

Glenda A Balderson

Data Manager $^{2}$

Stephen Munn

MB ChB, FRACs,

Transplant Surgeon

John W Chen

MB BS, FRACS, PhD

Director $^{4}$

Michael A Fink

MB BS, FRACS,

Lecturer $^{5}$

Michael D Crawford

MB BS, FRACS, MMed, Head of Liver Transplant Surgery

Geoffrey W McCaughan MB BS, PhD, FRACP Hepatologist

1 Transplantation Services, Royal Prince Alfred Hospital, Sydney, NSW.

2 Australia and New Zealand Live Transplant Registry, Princess Alexandra Hospital, Brisbane, QLD.

3 New Zealand Live Transplant Unit, Auckland City Hospital, Auckland, New Zealand

4 South Australian Live Transplant Unit,

Flinders Medical Centre Adelaide, SA.

5 Department of Surgery, University of Melbourne, Austin Health Melbourne, VIC

6 AW Morrow Gastroenterology and Live Centre, Royal Prince Alfred Hospital, Alfred Hospital,
Sydney, NSW.

verran@ausdoctors.net

MJA 2013; 199: 610-612 doi: 10.5694/mjal3.10102

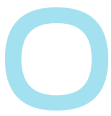

rthotopic liver transplantation (OLTx) has become an established treatment modality for managing end-stage liver disease, ${ }^{1}$ with the rates of OLTx in Australasia reflecting the rates of organ donation. ${ }^{2}$ Because lifelong immunosuppressive drug therapy is required for virtually all patients who have had OLTx, complications of immunosuppression, including the increased risk of malignancy, need to be addressed after transplantation. ${ }^{3,4}$

Over the past decade, it has become apparent that the incidence of malignancy for all solid organ transplant recipients is twofold higher than for the general population..$^{5}$ For patients who have undergone OLTx, de-novo malignancy is increasingly a significant cause of late morbidity and mortality after transplantation. ${ }^{3}$ However, the incidence and types of denovo malignancy that occur in the post-transplant phase vary according to the transplanted organ. ${ }^{5}$

One malignancy for which increased incidence after liver transplantation has been reported is colorectal malignancy. ${ }^{4,6,7}$ In a recent study of Australian transplant recipients, the incidence of colorectal malignancy was elevated among patients who had undergone OLTx compared with the general population (standardised incidence ratio $[S I R], 2.40) .{ }^{4}$ Such an increase was seen in lung transplant recipients but not in heart transplant recipients. ${ }^{4}$ However, the study did not examine overall recipient outcomes, such as mortality, or timing of cancer diagnoses. Also, a further analysis revealed no excess risk of mortality from de-novo colorectal malignancy in Australian patients who had undergone OLTx. ${ }^{8}$

We aimed to ascertain the nature and outcomes of colorectal malignancy diagnosed in the Australasian OLTx population from 1985 to 2011 a larger recipient cohort and a longer time frame than previously studied.

Objectives: To examine whether incidence of colorectal malignancy is increased in Australasian liver transplant recipients compared with the general population of Australia, and to assess the characteristics and outcomes of colorectal malignancy in this patient group.

Design, setting and patients: Data on patients who underwent orthotopic liver transplantation (OLTX) and had a diagnosis of de-novo colorectal malignancy after transplantation during the period 1985-2011 were obtained from the Australia and New Zealand Liver Transplant Registry, and these data were compared with colorectal malignancy data from the Australian Institute of Health and Welfare.

Main outcome measures: Time from OLTx to diagnosis of colorectal malignancy, stage of colorectal malignancy at diagnosis, patient survival, and standardised incidence ratio (SIR) for colorectal malignancy.

Results: Forty-eight of 3735 recipients (1.3\%) were diagnosed with colorectal malignancy at a median of 7.3 years after OLTX. More advanced colorectal malignancy (regional or metastatic disease) was evident at diagnosis in 20 of the 48 patients; these patients tended to be younger than patients with less advanced malignancy $(P=0.01)$ and diagnosed sooner after OLTX $(P=0.005)$. Despite treatment predominantly with surgery, 19 of the 48 patients died from the malignancy. The overall SIR for colorectal malignancy liver transplant recipients compared with the general population of Australia was $2.80(95 \% \mathrm{Cl}$, 2.06-3.71).

Conclusions: The incidence of colorectal malignancy is increased in liver transplant recipients in comparison with the general population. Of concern is the tendency for advanced malignancy to be diagnosed in younger patients. These data highlight the importance of considering whether specific guidelines for colorectal malignancy screening in the Australasian adult liver transplant population are needed.

\section{Methods}

Patients who had undergone OLTx and had a de-novo non-skin malignancy after transplantation during the period 1985-2011 were identified from data held by the Australia and New Zealand Liver Transplant Registry (ANZLTR). Data on the subset of recipients with colorectal malignancy were then extracted, including patient demographics, indications for transplantation, time of diagnosis of malignancy after transplantation, stage and treatment of malignancy and patient outcomes. The incidence of colorectal malignancy in Australian and New Zealand patients who underwent OLTx was determined using ANZLTR data. The incidence of colorectal malignancy in the general population of Australia was obtained from the Australian Institute of Health and Welfare's Australian Cancer Incidence and Mortality data books. ${ }^{9}$ SIRs for colorectal malignancy in the OLTx population compared with the general population were calculated by dividing the observed number of cases by the expected number of cases using the New York State Department of Health calculator. ${ }^{10}$ Other tests were performed using PASW Statistics 18 (SPSS Inc). The oversight committee for the ANZLTR approved the study.

\section{Results}

Two hundred and fifty-seven of 3735 patients who had undergone OLTx (6.9\%) developed de-novo non-skin malignancy, and 48 of them (1.3\%) were diagnosed with colorectal malignancy. The demographics of those with colorectal malignancy are shown in Box 1. There was a predominance of male recipients and the overall median age at diagnosis was 59 years. The most common underlying indica- 
tion for OLTx was liver disease due to primary sclerosing cholangitis, followed by liver disease due to alcohol and liver disease due to hepatitis $\mathrm{C}$. The median time to diagnosis of colorectal malignancy after OLTx was 87.6 months ( 7.3 years).

Just over half of the patients with colorectal malignancy had localised disease on presentation, and the remainder had more advanced disease (Box 2). Patients who were diagnosed when they had more advanced disease were younger $(P=0.01)$ and received the diagnosis sooner after OLTx $(P=0.005)$ compared with patients who were diagnosed when they had localised malignancy. Treatment was predominantly surgical in the first instance. The mortality rate due to colorectal malignancy was moderately high (19 patients died from malignancy), and it increased as the stage at time of diagnosis became more advanced $(P<0.001)$. Median survival of patients who had metastatic disease at diagnosis was 2 months (range, 0.25-15 months).

The incidence of colorectal malignancy among patients who had undergone OLTx was higher than expected, with an SIR of 2.80 compared with the general population of Australia (Box 3). In the 45-65-year age group, the SIR was very much elevated compared with the $>65$-year age group. As there were only two cases of colorectal malignancy recorded after OLTx in the $<45$-year age group, data for this group were excluded from the calculation for the overall SIR.

\section{Discussion}

This study shows that during the period 1985-2011, the risk of colorectal malignancy after liver transplantation was increased. The diagnosis was made in $1.3 \%$ of patients in Australasia who had undergone OLTx.

An apparent association between colorectal malignancy and one of the underlying disease indications for liver transplantation, liver disease due to primary sclerosing cholangitis, has been observed. ${ }^{6,7}$ This is thought to be related to the increased incidence of colorectal malignancy in patients who have both primary sclerosing cholangitis and ulcerative colitis. ${ }^{7}$ Our study also showed a tendency for colorectal malignancy to occur in younger patients, which has been shown in previous studies, ${ }^{11,12}$ including a study of patients in the Israel Penn International Transplant Tumor Registry. ${ }^{13}$

Our finding that the incidence of colorectal malignancy is increased in patients who have undergone OLTx compared with the general population of Australia (SIR, 2.80) mirrors the previously reported finding of an overall SIR of 2.40. ${ }^{4}$ Similarly, an SIR of 2.16 has been reported from a European liver transplant program ${ }^{6}$ and an SIR of 2.56 has been calculated using pooled data from a systematic review. ${ }^{7}$ This increased risk adds to the burden of colorectal malignancy, which is likely to soon become the second most commonly diagnosed malignancy in the general population of Australia and New Zealand. ${ }^{9,14}$

Also of concern is the high proportion of patients in our study who presented with advanced or disseminated malignancy, as reflected in the mortality rate. The tendency for liver transplant recipients to present with
1 Demographics of Australasian liver transplant recipients diagnosed with colorectal malignancy $(n=48)$

\begin{tabular}{lc} 
& No. or median \\
\hline Male & 34 \\
Cause of underlying liver disease & 17 \\
Primary sclerosing cholangitis & 5 \\
Primary biliary cirrhosis & 8 \\
Alcohol & 4 \\
Hepatitis B & 7 \\
Hepatitis C & 1 \\
Hepatoblastoma & 6 \\
Other & 59 (12-78) \\
Median age at time diagnosis of & \\
malignancy, years (range) & \\
Median time from orthotopic liver & \\
transplantation to diagnosis, months & \\
(range) &
\end{tabular}

more advanced colorectal malignancy that leads to death has also been noted in other reports, ${ }^{12,13}$ but not in a previous Australasian study. ${ }^{8}$ The additional 5 years of registry followup data underpinning our study, compared with the previous Australian study, ${ }^{8}$ may explain this difference. Furthermore, advanced malignancy tended to be diagnosed in younger
2 Demographics, presentation, treatment and outcome of patients with colorectal malignancy following liver transplantation, analysed according to stage at diagnosis*

\begin{tabular}{|c|c|c|c|c|}
\hline & \multicolumn{3}{|c|}{ Stage } & \multirow[b]{2}{*}{$P$} \\
\hline & Localised $(n=28)$ & Regional $(n=6)$ & Metastatic $(n=14)$ & \\
\hline Male & 18 & 5 & 11 & 0.003 \\
\hline Median age at diagnosis, years (range) & $64(54-78)$ & $55(47-61)$ & $57(12-69)$ & 0.01 \\
\hline Median time from orthotopic liver & $107.9(9.2-207.9)$ & $31.1(3.0-57.7)$ & $71.2(12.6-147.5)$ & 0.005 \\
\hline
\end{tabular}

transplantation to diagnosis, months (range)

Cause of underlying liver disease

0.006

Primary sclerosing cholangitis

Primary biliary cirrhosis

Alcohol

Hepatitis B

Hepatitis C

Hepatoblastoma

Other

Location of malignancy

Colon

Rectum

Initial treatment

Surgery

Chemotherapy

Radiotherapy

Palliation

Outcome

Alive at study completion date

Died from malignancy

Died from other cause

* Data are number unless otherwise specified. 
3 Standardised incidence ratio for colorectal malignancy in Australasian liver transplant recipients compared with general population of Australia, by age group

\begin{tabular}{lccc} 
Age group & $\begin{array}{c}\text { No. of } \\
\text { observed cases }\end{array}$ & $\begin{array}{c}\text { No. of } \\
\text { expected cases }\end{array}$ & $\begin{array}{c}\text { Standardised incidence ratio } \\
(95 \% \mathrm{CI})\end{array}$ \\
\hline$<45$ years & 2 & 0.4 & - \\
$45-65$ years & 35 & 12 & $15.46(1.23-18.48)$ \\
$>$ 65 years & 11 & 20 & $1.58(0.004-1.73)$ \\
Overall & 48 & 17 & $2.80(2.06-3.71) *$ \\
\hline * Data for the < 45-year age group were excluded from the calculation for the overall SIR.
\end{tabular}

patients and was diagnosed soon after transplantation (median time from OLTx to diagnosis, $<6$ years).

As data on screening for colorectal malignancy are not held by the ANZLTR, it was not possible to ascertain whether screening was performed before or after liver transplantation. Liver transplant recipients are now screened for colorectal malignancy in other regions of the world to achieve early diagnoses ${ }^{6}$ and improve survival. ${ }^{15}$ Screening for colorectal malignancy after liver transplantation has been shown to increase the detection of neoplastic polyps. ${ }^{16,17}$ In the Australasian context, a trial of screening for colorectal cancer in kidney transplant recipients led to the detection of colorectal neoplasia in $13 \%$ of participants. ${ }^{18}$

These data highlight the importance of considering whether specific guidelines for colorectal malignancy screening in the Australasian adult liver transplant population are needed. Recommendations range from screening all liver transplant patients whose underlying liver disease is due to primary sclerosing cholangitis ${ }^{7}$ every 2 years to undertaking routine screening as per local guidelines for the general population. ${ }^{19}$ Continual examination of registry data to help formulate iterations of screening guidelines is also important.

Competing interests: No relevant disclosures. Received 21 Jan 2013, accepted 16 Jun 2013.

1 Dienstag JL, Cosimi AB. Liver transplantation a

2 Prakoso E, Verran D, Dilworth P, et al. Increasing liver transplantation waiting list mortality: a report from the Australian National Liver Transplantation Unit, Sydney. Intern Med J 2010; 40: 619-625.

3 Gelson W, Hoare M, Dawwas MF, et al. The pattern of late mortality in liver transplant recipients in the United Kingdom. Transplantation 2011; 91: 1240-1244.

$4 \mathrm{Na} \mathrm{R}$, Grulich AE, Meagher NS, et al. Comparison of de novo cancer incidence in Australian liver, heart and lung recipients. Am J Transplant 2013; 13: 174-183.

5 Engels EA, Pfeiffer RM, Fraumeni JF, et al. Spectrum of cancer risk among US solid organ transplant recipients. JAMA 2011; 306: 18911901.

6 Sint Nicolaas J, Tjon AS, Metselaar HJ, et al. Colorectal cancer in post-liver transplant recipients. Dis Colon Rectum 2010; 53: 817-821.

7 Sint Nicolaas J, de Jonge V, Steyerberg EW, et al. Risk of colorectal carcinoma in post-liver meta-analysis. Am J Transplant 2010; 10 : 868-876.

$8 \mathrm{Na} \mathrm{R}$, Grulich AE, Meagher NS, et al. De novo cancer-related death in Australian liver and vision realized. N Engl J Med 2012; 367: 1483-1485. transplant patients: a systematic review and cardiothoracic transplant recipients. Am J Transplant 2013; 13: 1296-1304.

9 Australian Institute of Health and Welfare. Australian Cancer Incidence and Mortality (ACIM) books. http://www.aihw.gov.au/acimbooks (accessed Jan 2013).

10 New York State Department of Health. Guidance for health outcome data review and analysis relating to NYSDEC environmental justice and permitting: calculation of confidence intervals using cancer data from the NYSDOH website. http://www.health.ny.gov/ environmental/investigations/environmental justice/hod/docs/cancer_data_example.xls (accessed Oct 2013).

11 Chak E, Saab S. Risk factors and incidence of de novo malignancy in liver transplant recipients: a systematic review. Liver Int 2010; 30: 1247-1258.

12 Johnson EE, Leverson GE, Prisch JD, Heise CP. A 30-year analysis of colorectal adenocarcinoma in transplant recipients and proposal for altered screening. J Gastrointest Surg 2007; 11: 272-279.

13 Buell JF, Papaconstantinou HT, Skalow B, et al. De novo colorectal cancer: five-year survival is markedly lower in transplant recipients compared with the general population. Transplant Proc 2005; 37: 960-961.

14 Ministry of Health, New Zealand. Cancer data and stats: statistical publications and data sets on cancer. http://www.health.govt.nz/nzhealth-statistics/health-statistics-and-datasets/cancer-data-and-stats?mega=Health\% 20statistics\&title=Cancer (accessed Jan 2013).

15 Finkenstedt A, Graziadei IW, Oberaigner W, et al. Extensive surveillance promotes early diagnosis and improved survival of de novo malignancies in liver transplant recipients. Am J Transplant 2009; 9: 2355-2361.

16 Park HY, Chang BY, Lim SW, et al. Risk of colorectal neoplasia in patients with solid organ transplantation. Clin Transplant 2012; 2650-2656.

17 Albright JB, Bonatti H, Stauffer J, et al. Colorectal and anal neoplasms following liver transplantation. Colorectal Dis 2010; 12 657-666.

18 Collins MG, Teo E, Cole SR, et al. Screening for colorectal cancer and advanced colorectal neoplasia in kidney transplant recipients: cross sectional prevalence and diagnostic accuracy study of faecal immunochemical testing for haemoglobin and colonoscopy. BMJ 2012; 345: e4657.

19 Herrero I. Screening of de novo tumours after liver transplantation. J Gastroenterol Hepatol 2012; 27: 1011-1016. 